\title{
Time and spectrally resolved enhanced fluorescence using silver nanoparticle impregnated polycarbonate substrates
}

\author{
Laura Lagonigro, ${ }^{1}$ Anna C. Peacock, ${ }^{1, a)}$ Stefan Rohrmoser, ${ }^{2}$ Tom Hasell, $^{3}$ \\ Steven M. Howdle, ${ }^{3}$ Pier J. A. Sazio, ${ }^{1}$ and Pavlos G. Lagoudakis ${ }^{2}$ \\ ${ }^{1}$ Optoelectronics Research Centre, University of Southampton, Southampton SO17 1BJ, United Kingdom \\ ${ }^{2}$ School of Physics and Astronomy, University of Southampton, Southampton SO17 1BJ, \\ United Kingdom \\ ${ }^{3}$ School of Chemistry, University of Nottingham, Nottingham NG7 2RD, United Kingdom
}

(Received 1 August 2008; accepted 3 December 2008; published online 31 December 2008)

\begin{abstract}
Silver nanoparticle impregnated polycarbonate strips have been investigated as substrates for metal-enhanced photoluminescence of a blue emitting dye molecule (coumarin 102). By considering simultaneous time and spectrally resolved photoluminescence we observed fluorescence enhancement resulting from plasmon coupling with an increase in the emission by a factor of $\sim 8.5$ with an associated reduction in the photon lifetime. We relate the fast and slow components of the observed emission decay to the presence of both monomers and aggregates in the films and we discuss their different responses to the plasmon coupling. () 2008 American Institute of Physics. [DOI: $10.1063 / 1.3059567]$
\end{abstract}

Photoluminescence (PL) plays a central role in many aspects of biological and medical research with applications including DNA sequencing, ${ }^{1}$ cell imaging, ${ }^{2}$ and sensing. ${ }^{3}$ Recently there has been much interest in modifying the photonic mode density of states around the fluorescent molecule using surface plasmon excitation on metal nanoparticles. ${ }^{4}$ The large electric fields associated with plasmon modes can enhance photon-matter interaction to modify the excitation cross section ${ }^{5}$ and the radiative decay rate, resulting in increased fluorescence intensities of molecules in close proximity to the metal. ${ }^{6}$ Metal-enhanced fluorescence (MEF) has led to the demonstration of optical probes with enhanced brightness so that we expect it to play an important role in the next generation of photonic devices. ${ }^{4}$

If MEF is to be used readily in routine procedures, then ideally the substrates need to be robust, stable, and most importantly, biocompatible. To date, typical substrates employed for MEF were similar to those used for other opticalplasmonic applications such as surface enhanced Raman scattering (SERS) spectroscopy. ${ }^{7}$ Conventional processing techniques for the fabrication of planar SERS substrates are often expensive and in general have the active metal surface exposed to air so that they not only suffer from poor temporal stability, resulting from oxidation, but are also fragile. We have recently reported substrates fabricated via a supercritical technique where metal nanoparticles are embedded into polymer substrates that were shown to exhibit an efficient SERS response. ${ }^{8}$ The nanoparticle composites are fabricated using polycarbonate as the host polymer, which has excellent biocompatibility, as well as superior optical and mechanical properties. ${ }^{9}$ These composite structures offer a number of advantages over previously reported metal-polymer substrates fabricated via surface functionalization ${ }^{10}$ in that by embedding the nanoparticles in the polymer they are protected from the surrounding environment, thus yielding a high degree of temporal stability. In this paper we investigate the MEF properties of these substrates and show them to be

${ }^{\text {a)} E l e c t r o n i c ~ m a i l: ~ a c p @ o r c . s o t o n . a c . u k . ~}$ excellent candidates for light emitting applications.

The silver nanoparticles are synthesized in situ within the polycarbonate strips using a high-pressure processing technique that diffuses an organometallic precursor into the hosting material ${ }^{11}$ via a supercritical carbon dioxide $\left(\mathrm{scCO}_{2}\right)$ solvent, as reported in detail in Ref. 8. Transmission electron microscopy (TEM) and energy dispersive characterization of the silver impregnated polymer substrates confirmed the presence of a homogeneous band of metallic silver nanoparticles located along the edge of the polycarbonate strips with a size distribution of $\sim 2-10 \mathrm{~nm}$ in diameter (see supplementary information in Ref. 8). To illustrate the uniformity of the nanoparticle films, Fig. 1(a) shows a TEM micrograph of the cross section of a silver-polycarbonate substrate $(\sim 100 \mathrm{~nm}$ thick) where we can see a precisely defined film thickness of $\sim 6.5 \mu \mathrm{m}$. The inset shows a closeup of the silver particles on the outside edge of the polycarbonate. As MEF is a highly localized effect, acting over nanometer length scales, only the particles situated in close proximity to the surface will contribute to the fluorescence enhancement of molecules deposited on the substrates. The measured UV-visible extinction spectrum of the silver-polycarbonate substrate (solid line) is shown in Fig. 1(b), together with a digital photograph
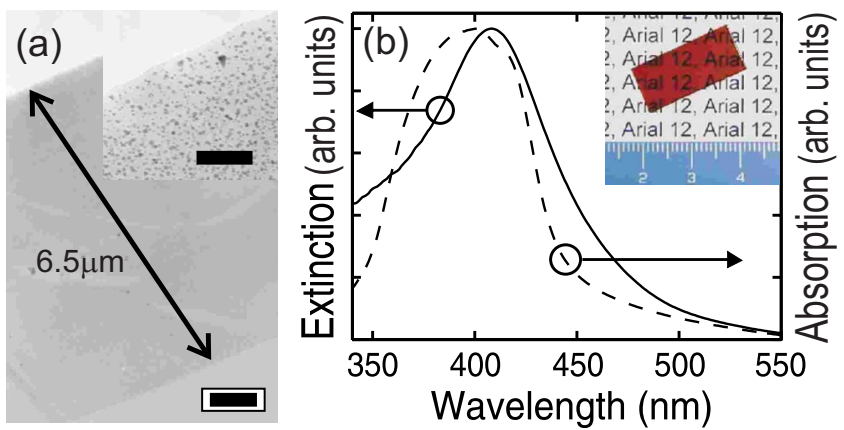

FIG. 1. (Color online) (a) TEM image of AgPC substrate (scale bar: $1 \mu \mathrm{m}$ ) Inset: closeup of surface nanoparticles (scale bar: $200 \mathrm{~nm}$ ). (b) Normalized extinction of AgPC (solid) and absorption of coumarin on PC (dashed) Inset: photograph of AgPC substrate. 
of the substrate to highlight the transparency and the homogeneity of the silver films. The plasmonic peak is centered around $408 \mathrm{~nm}$ and is relatively broad due to the nanoparticle size dispersion. The position of the peak is in excellent agreement with the predictions of Mie scattering theory ${ }^{12}$ for particles with diameters $<10 \mathrm{~nm}$ embedded in a dielectric host with a refractive index of the pure polycarbonate $n$ $=1.585$ (mean peak position $\sim 411 \mathrm{~nm}$ ), providing further confirmation of the TEM size estimates.

To test the metal-polymer composites for MEF we prepared the silver-modified (AgPC) and pure polycarbonate $(\mathrm{PC})$ substrates by spin coating a weak $0.5 \mathrm{mM}$ solution of coumarin $102(2,3,5,6-1 H, 4 H$-tetrahydro8-methylquinolazin-[9,9a,1-gh]), purchased from Radiant Dyes Laser \& Accessories $\mathrm{GmbH}$, in ethanol at $3000 \mathrm{rpm}$ for $1 \mathrm{~min}$. The deposition conditions were monitored in order to reproduce the same solution film thickness for both samples. For comparison, the normalized absorption spectrum of coumarin on the PC substrate is plotted with the AgPC extinction spectrum in Fig. 1(b) (dashed line), showing a strong spectral overlap so that we expect an efficient coupling between the plasmon modes excited on the metal nanoparticles and fluorescing dye molecules.

Confirmation of MEF can be provided through an observed reduction in the photon lifetime using simultaneous time and spectral resolved measurements. To investigate this, the samples were excited with a frequency-doubled modelocked Ti:sapphire laser (400 nm excitation wavelength, $\sim 120$ fs pulse width, $80 \mathrm{MHz}$ repetition rate with an average power of $1.5 \mathrm{~mW}$, and a spot size of $30 \mu \mathrm{m}$ ), resonant with the plasmonic peak of the AgPC substrate. The emitted fluorescence from the dye molecules was collected in reflection, coupled into a multimode fiber, and analyzed by a synchroscan streak camera (Hamamatsu C5680) with a time resolution of $25 \mathrm{ps}$ coupled to the exit of a $25 \mathrm{~cm}$ monochromator with a $300 \mathrm{gr} / \mathrm{mm}$ grating.

Preliminary photon lifetime measurements were performed on both high $(\sim 0.04 \mathrm{M}$ saturated solution) and low (1000 times diluted) concentration solutions of coumarin in ethanol. In both cases the photon lifetime decay curves could be fitted with a single exponential decay, with photon lifetimes equal to $\tau_{H}=364 \pm 3$ and $\tau_{L}=452 \pm 3 \mathrm{ps}$, respectively. The faster decay of the high concentration solution is expected as in saturated solutions the intermolecular interactions introduce deactivation channels. In addition, when the dye molecules aggregate then a further reduction in lifetime will occur due to the formation of $J$-band excitons, as observed in thin (even down to monolayer coverage) film depositions, ${ }^{13}$ so that we anticipate the dye molecules to exhibit an even shorter lifetime once deposited on the substrates.

Time-spectral resolved PL measurements were then performed on both the (a) PC and (b) AgPC substrates with the resulting profiles shown in Fig. 2. From these we can obtain both the time integrated PL spectra (c) and the averaged PL decay curves (d) for the wavelength range of 438-468 nm where the emission was greatest. Comparing the PL spectra we observe that while both profiles have the same shape, the emission intensity is $\sim 8.5$ times higher for coumarin when deposited on the AgPC substrate, an enhancement of similar magnitude to those reported elsewhere in literature. ${ }^{10}$ Furthermore, from the decay curves it is clear that this enhanced
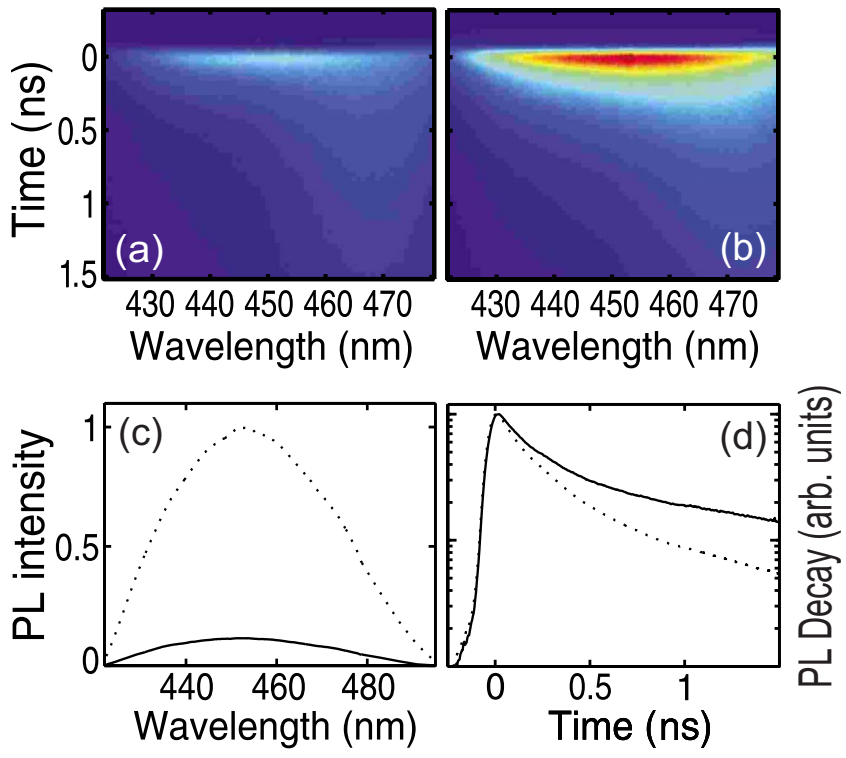

FIG. 2. (Color online) Time and spectrally resolved PL for coumarin on (a) PC and (b) AgPC substrates. (c) Integrated PL spectra and (d) average PL decays on PC (solid) and AgPC (dotted).

fluorescence is accompanied by a reduction in the photon lifetime.

With the PL decay profiles plotted on a logarithmic scale, it is clear that the coumarin films on both the AgPC and PC substrates are now characterized by a biexponential behavior. From our previous observations, we attribute this behavior to the coexistence of aggregates, as the fast species, and monomers, as the slow species, in the films. Fitting these curves with double exponentials to obtain both the fast $\left(\tau_{1}\right)$ and slow $\left(\tau_{2}\right)$ components we obtained $\tau_{1}=172 \pm 2$ and $\tau_{2}$ $=1627 \pm 37 \mathrm{ps}$ for the PC substrate and the modified lifetimes of $\tau_{1}^{*}=154 \pm 3$ and $\tau_{2}^{*}=889 \pm 26$ ps for the AgPC substrate. While in both cases there is an observed reduction in lifetime, it is clear that the lifetime of the monomers is more greatly affected by the presence of the Ag. Calculating the Purcell enhancement factors ${ }^{14} F$ for the two species yields $F_{1}=\tau_{1} / \tau_{1}^{*}=1.12 \pm 0.03$ and $F_{2}=\tau_{2} / \tau_{2}^{*}=1.83 \pm 0.10$. This difference in the enhancement factors can be attributed to a delocalization of the excitonic dipole in the aggregates, ${ }^{15}$ so that the overlap with the plasmon enhanced field is reduced for the aggregates compared with the monomers. The small Purcell factors compared to the large ( $~ 8.5$ times) PL enhancement suggest that the increased fluorescence is not only due to a modification of the radiative channels, but that additional factors, such as an increase in the absorption cross section, ${ }^{16}$ also need to be considered.

Further to this, we have also investigated the emission rates, where $k=1 / \tau$, and Purcell factors as functions of wavelength. Figure 3 shows both the (a) fast and (b) slow components of the photon emission rate for the two substrates, as calculated from the time-spectral profiles in Figs. 2(a) and 2(b) for selected wavelengths over the emission peak. The corresponding Purcell factor $F(\lambda)=k^{*}(\lambda) / k(\lambda)$ is then plotted in Fig. 3(c), where we see that the fast and slow components show a different response to the surface plasmon excitation as a function of wavelength. In particular, the rate enhancement $F_{2}(\lambda)$ of the slow component, associated with the dye monomers, exhibits an oscillatory behavior, which is similar to the results previously reported by Okamoto et al. ${ }^{17}$ How- 


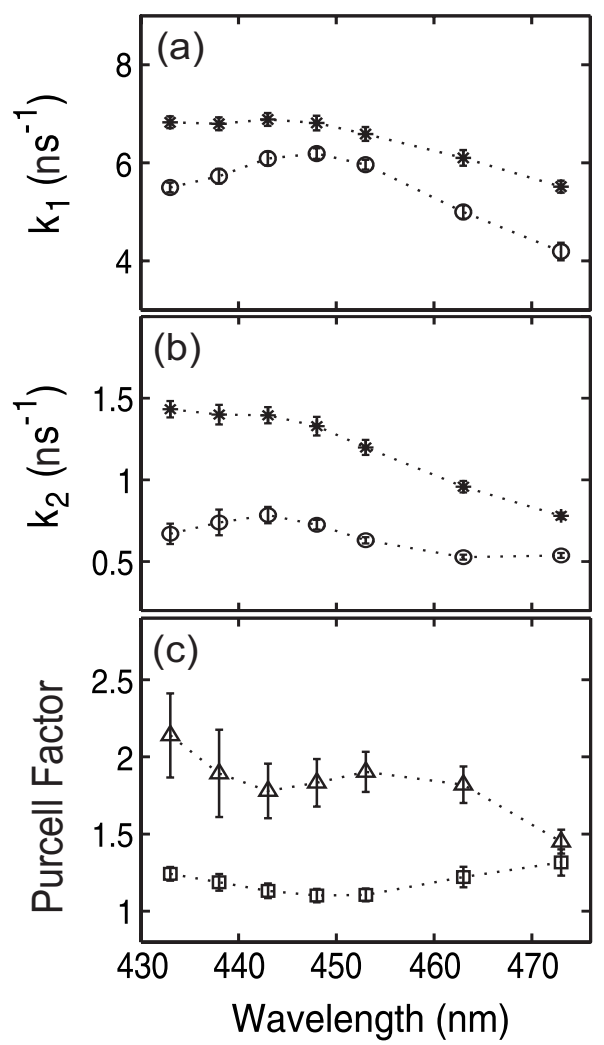

FIG. 3. (a) Fast and (b) slow components of the photon emission rate for coumarin on PC (circle) and AgPC (star) as functions of wavelength; data points averaged over $\pm 5 \mathrm{~nm}$. (c) Purcell factor for the fast (square) and slow (triangle) components.

ever, the fast component $F_{1}(\lambda)$ appears to only exhibit a minimum around the emission peak at $450 \mathrm{~nm}$, though this could also be due to an oscillatory behavior on a different wavelength scale.

In summary, we report the use of silver impregnated polymer substrates as enhanced fluorescence probes. These composite films combine the excellent plasmonic properties of silver nanoparticles to yield large electric fields in the vicinity of the fluorophore, with the convenience of a robust, biocompatible polymer host matrix. MEF of coumarin 102 on the silver-polycarbonate substrates was demonstrated using simultaneous time and spectrally resolved PL. Owing to the versatility of this supercritical technique and its potential to be employed with a range of host polymer matrices and metal precursors, these substrates can be readily tailored for a wide range of applications in medicine and biology.

This work was supported by EPSRC-GB (Grant No. EP/ F013876/1), the University of Southampton Annual Adventure in Research Grant No. A2005/18, and A.C.P. holds a Royal Academy of Engineering fellowship.

${ }^{1}$ L. M. Smith, J. Z. Sanders, R. J. Kaiser, P. Hughes, C. Dodd, C. R. Connell, C. Heiner, S. B. H. Kent, and L. E. Hood, Nature (London) 321, 674 (1986).

${ }^{2}$ D. J. Stephens and V. J. Allan, Science 300, 82 (2003).

${ }^{3}$ P. K. Jain, X. Huang, I. H. El-Sayed, and M. A. El-Sayad, Plasmonics 2, 107 (2007).

${ }^{4}$ J. R. Lakowicz, J. Malicka, I. Gryczynski, Z. Gryczynski, and C. D. Geddes, J. Phys. D 36, R240 (2003).

${ }^{5}$ J. S. Biteen, D. Pacifici, N. S. Lewis, and H. A. Atwater, Nano Lett. 5, 1768 (2005)

${ }^{6}$ J. R. Lakowicz, Anal. Biochem. 337, 171 (2005).

${ }^{7}$ D. S. dos Santos Jr. and R. F. Aroca, Analyst (Cambridge, U.K.) 132, 450 (2007).

${ }^{8}$ T. Hasell, L. Lagonigro, A. C. Peacock, S. Yoda, P. D. Brown, P. J. A. Sazio, and S. M. Howdle, Adv. Funct. Mater. 18, 1265 (2008).

${ }^{9}$ K. Aslan, P. Holley, and C. D. Geddes, J. Mater. Chem. 16, 2846 (2006).

${ }^{10}$ K. Aslan, Z. Leonenko, J. R. Lakowicz, and C. D. Geddes, J. Fluoresc. 15, 643 (2005).

${ }^{11}$ T. Hasell, K. J. Thurecht, R. D. W. Jones, P. D. Brown, and S. M. Howdle, Chem. Commun. (Cambridge) 2007, 3933.

${ }^{12}$ C. F. Bohren, Absorption and Scattering of Light by Small Particles (Wiley, Weinheim, Germany, 1940).

${ }^{13}$ K. Ray, A. K. Dutta, and T. N. Misra, J. Lumin. 71, 123 (1997).

${ }^{14}$ M. Boroditsky, R. Vrijen, T. F. Krauss, R. Coccioli, R. Bhat, and E. Yablonovitch, J. Lightwave Technol. 17, 2096 (1999).

${ }^{15}$ P. G. Lagoudakis, M. M. de Souza, F. Schindler, J. M. Lupton, J. Feldmann, J. Wenus, and D. G. Lidzey, Phys. Rev. Lett. 93, 257401 (2004).

${ }^{16}$ J. S. Biteen, D. Pacifici, N. S. Lewis, and H. A. Atwater, Nano Lett. 5 , 2116 (2005); F. Tam, G. P. Goodrich, B. R. Johnson, and N. J. Halas, ibid. 7, 496 (2007).

${ }^{17}$ K. Okamoto, I. Niki, A. Scherer, Y. Narukawa, T. Mukai, and Y. Kawakami, Appl. Phys. Lett. 87, 071102 (2005). 